\title{
Canonical Bases and Piecewise-linear Combinatorics
}

\author{
Roger Carter \\ Mathematics Institute, University of Warwick, Coventry CV4 7AL, England \\ E-mail:rwc@maths.warwick.ac.uk \\ Robert Marsh \\ Department of Mathematics and Computer Science, University of Leicester, University Road, Leicester LE1 \\ $7 R H$, England \\ E-mail: R.Marsh@mcs.le.ac.uk
}

\begin{abstract}
Let $U_{q}$ be the quantum group associated to a Lie algebra $\mathbf{g}$ of rank $n$. The negative part $U_{q}^{-}$of $U_{q}$ has a canonical basis B with favourable properties (see Kashiwara [3] and Lusztig [6, §14.4.6]). The approaches of Lusztig and Kashiwara lead to a set of alternative parametrizations of the canonical basis, one for each reduced expression for the longest word in the Weyl group of $\mathbf{g}$. We describe the authors' recent work establishing close relationships between the Lusztig cones, canonical basis elements and the regions of linearity of reparametrization functions arising from the above parametrizations in type $A_{4}$ and give some speculations for type $A_{n}$.

Keywords: Quantum group, Lie algebra, Canonical basis, Tight monomials, Weyl group, Piecewise-linear functions.
\end{abstract}

\section{Introduction}

Let $\mathbf{g}$ be a finite-dimensional simple Lie algebra over $\mathbb{C}$ and $U_{q}(\mathbf{g})$ be the corresponding quantized enveloping algebra over $\mathbb{C}\left[v, v^{-1}\right]$. Let

$$
U_{q}(\mathbf{g})=U_{q}^{-} \otimes U_{q}^{0} \otimes U_{q}^{+}
$$

be the triangular decomposition of $U_{q}(\mathbf{g})$. Let $\mathbf{B}$ be the canonical basis of $U_{q}^{-}$, introduced independently by Lusztig and Kashiwara. It is natural to ask how the elements of $\mathbf{B}$ are expressed in terms of the generators $F_{1}, F_{2}, \ldots, F_{n}$ of $U_{q}^{-}$. This is a difficult question which is known completely in only a few low rank cases, but the attempt to understand it has led to a remarkable theory of piecewise-linear combinatorics associated with the canonical basis.

In this paper we consider only the case in which $\mathbf{g}$ has type $A_{n}$. We describe the situation for small values of $n$ before giving some speculations for arbitrary $n$.

First suppose that $\mathbf{g}$ has type $A_{1}$. We write:

$$
\begin{aligned}
\mathbb{N} & =\{a \in \mathbb{Z}: a \geq 0\} \\
{[a] } & =\frac{v^{a}-v^{-a}}{v-v^{-1}}, \quad \text { for } a \in \mathbb{N}, \\
{[a] ! } & =[1][2] \cdots[a], \text { for } a \in \mathbb{N},
\end{aligned}
$$


and $F_{1}^{(a)}=F_{1}^{a} /[a] !$

It was shown by Lusztig 晒 that $\mathbf{B}=\left\{F_{1}^{(a)}: a \in \mathbb{N}\right\}$. Thus the canonical basis elements are the quantized divided powers of the generator $F_{1}$.

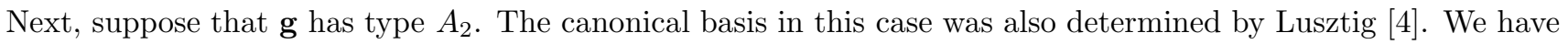

$$
\begin{aligned}
\mathbf{B}=\quad & \left\{F_{1}^{(a)} F_{2}^{(b)} F_{1}^{(c)}: a, b, c \in \mathbb{N}, b \geq a+c\right\} \\
& \cup\left\{F_{2}^{(a)} F_{1}^{(b)} F_{2}^{(c)}: a, b, c \in \mathbb{N}, b \geq a+c\right\} .
\end{aligned}
$$

When $b=a+c$ one has the relation

$$
F_{1}^{(a)} F_{2}^{(a+c)} F_{1}^{(c)}=F_{2}^{(c)} F_{1}^{(a+c)} F_{2}^{(a)},
$$

but apart from this the above elements of $\mathbf{B}$ are all distinct. Thus in this case each canonical basis element may be written as a monomial in the generators $F_{1}, F_{2}$. The two types of monomials which arise are related to the two reduced decompositions of the longest element $w_{0}$ of the Weyl group $W$ of $\mathbf{g}$. We have $W=\left\langle s_{1}, s_{2}\right\rangle$ and $w_{0}=s_{1} s_{2} s_{1}=s_{2} s_{1} s_{2}$. Each of these reduced words for $w_{0}$ gives rise to a type of monomial in the canonical basis.

We now turn to the case when $\mathbf{g}$ has type $A_{3}$. Lusztig [5] obtained many, but not all, elements of $\mathbf{B}$ as monomials in the generators $F_{1}, F_{2}, F_{3}$ and gave an example of an element of $\mathbf{B}$ which could not be written as a monomial in $F_{1}, F_{2}, F_{3}$. The remaining elements of $\mathbf{B}$ were determined by Xi [10]. In order to describe the monomials in $\mathbf{B}$ we consider reduced words for $w_{0}$. In type $A_{3} w_{0}$ has 16 reduced expressions. However it is natural to divide them into equivalence classes called commutation classes; two reduced words being equivalent if one can be obtained from the other by a succession of commutations in the Coxeter generators of $W$. For example, $s_{1} s_{3} s_{2} s_{1} s_{3} s_{2}$ is in the same commutation class as $s_{3} s_{1} s_{2} s_{3} s_{1} s_{2}$, where the generators are numbered as in the Dynkin diagram (see Figure 1).

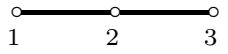

Figure 1: Dynkin diagram of type $A_{3}$.

There are 8 commutation classes of reduced words for $w_{0}$ in type $A_{3}$. Each of these gives rise to a family of monomials in $F_{1}, F_{2}, F_{3}$ which lie in $\mathbf{B}$. For example, the reduced word $w_{0}=s_{1} s_{3} s_{2} s_{1} s_{3} s_{2}$ gives rise to monomials

$$
F_{1}^{(a)} F_{3}^{(b)} F_{2}^{(c)} F_{1}^{(d)} F_{3}^{(e)} F_{2}^{(f)}, \quad a, b, c, d, e, f \in \mathbb{N},
$$

and such a monomial was shown by Lusztig to lie in the canonical basis $\mathbf{B}$ provided

$$
c \geq a+d, \quad c \geq b+e, \quad d+e \geq c+f .
$$

These inequalities come from considering two consecutive occurrences of a given Coxeter generator $s_{i}$ in the given reduced word. The sum of the exponents corresponding to these occurrences of $s_{i}$ is less than or equal to the sum of the exponents corresponding to Coxeter generators $s_{j}$ between these two occurrences of $s_{i}$ such that $s_{j}$ does not commute with $s_{i}$. Lusztig obtained 8 families of monomials in $\mathbf{B}$ in this way, and the remaining elements of $\mathbf{B}$ determined by Xi are linear combinations of monomials with coefficients which are quantum binomial coefficients. 
We now suppose that $\mathbf{g}$ has type $A_{4}$. Here the situation is more involved and has been investigated by the authors. We shall outline the situation in the present paper, and hope to publish the proofs in a subsequent article. For each reduced word $w_{0}=s_{i_{1}} s_{i_{2}} \cdots s_{i_{k}}$, where $k=\ell\left(w_{0}\right)$, we write $\mathbf{i}=\left(i_{1}, i_{2}, \ldots, i_{k}\right)$. For each such $\mathbf{i}$, we define a subset $C_{\mathbf{i}}$ of $\mathbb{N}^{k}$ whose definition is motivated by the rule mentioned above for a monomial to lie in $\mathbf{B}$ in type $A_{3}$. We define $C_{\mathbf{i}}$ to be the set of those $\mathbf{a} \in \mathbb{N}^{k}$ such that for each pair $t, t^{\prime} \in[1, k]$ with $t<t^{\prime}, i_{t}=i_{t^{\prime}}, i_{p} \neq i_{t}$ for $t<p<t^{\prime}$, we have $\sum_{p} a_{p} \geq a_{t}+a_{t^{\prime}}$, summed over all $p$ with $t<p<t^{\prime}$ such that $s_{p}$ does not commute with $s_{i_{t}}$.

The cone $C_{\mathbf{i}}$ will be called the Lusztig cone associated with $\mathbf{i}$. The elements of $C_{\mathbf{i}}$ give rise to monomials. Let

$$
M_{\mathbf{i}}=\left\{F_{i_{1}}^{\left(a_{1}\right)} F_{i_{2}}^{\left(a_{2}\right)} \cdots F_{i_{k}}^{\left(a_{k}\right)}: \mathbf{a}=\left(a_{1}, a_{2}, \ldots, a_{k}\right) \in C_{\mathbf{i}}\right\} .
$$

It was shown by Marsh [7] that when $\mathbf{g}$ has type $A_{4}$ we have $M_{\mathbf{i}} \subseteq \mathbf{B}$ for each $\mathbf{i}$. In type $A_{4}$ there are 62 commutation classes of reduced words for $w_{0}$, and we obtain in this way 62 families of monomials in the canonical basis. These are far from being the only elements of $\mathbf{B}$, however, and the remaining elements are not known as expressions in terms of $F_{1}, F_{2}, F_{3}, F_{4}$.

\section{PBW-type Bases and the Canonical Basis}

We shall now recall Lusztig's approach to the canonical basis. For each reduced word $\mathbf{i}$ for $w_{0}$, Lusztig defined , a PBW-type basis $B_{\mathbf{i}}$ of $U_{q}^{-}$. The reduced word $w_{0}=s_{i_{1}} s_{i_{2}} \cdots s_{i_{k}}$ gives rise to a total order on the set $\Phi^{+}$of positive roots of $\mathbf{g}$. We have

$$
\Phi^{+}=\left\{\alpha^{1}, \alpha^{2}, \ldots, \alpha^{k}\right\}
$$

where

$$
\alpha^{1}=\alpha_{i_{1}}, \alpha^{2}=s_{i_{1}}\left(\alpha_{i_{2}}\right), \ldots \alpha^{k}=s_{i_{1}} s_{i_{2}} \cdots s_{i_{k-1}}\left(\alpha_{i_{k}}\right),
$$

and $\alpha_{1}, \alpha_{2}, \ldots, \alpha_{n}$ are the simple roots. By using braid group operations, Lusztig defines for each $\alpha \in \Phi^{+}$a root vector $F_{\alpha} \in U_{q}^{-}$, beginning with $F_{\alpha_{i}}=F_{i}$. We write $F_{\mathbf{i}}^{\mathbf{c}}=F_{\alpha^{1}}^{\left(c_{1}\right)} F_{\alpha^{2}}^{\left(c_{2}\right)} \cdots F_{\alpha^{k}}^{\left(c_{k}\right)}$ for $\mathbf{c}=\left(c_{1}, c_{2}, \ldots, c_{k}\right) \in \mathbb{N}^{k}$. Then the set $B_{\mathbf{i}}=\left\{F_{\mathbf{i}}^{\mathbf{c}}: \mathbf{c} \in \mathbb{N}^{k}\right\}$ is a basis for $U_{q}^{-}$, called the PBW-type basis associated to $\mathbf{i}$.

The lattice $\mathcal{L}=\mathbb{C}[v] B_{\mathbf{i}}$ was shown by Lusztig to be independent of $\mathbf{i}$ and there is a bijective map

$$
\begin{aligned}
\mathbf{B} & \longrightarrow B_{\mathbf{i}} \\
b & \mapsto F_{\mathbf{i}}^{\mathbf{c}}
\end{aligned}
$$

such that $b \equiv F_{\mathbf{i}}^{\mathbf{c}} \bmod v \mathcal{L}$. We write $\varphi_{\mathbf{i}}(b)=\mathbf{c}$. Then the map $\varphi_{\mathbf{i}}: \mathbf{B} \rightarrow \mathbb{N}^{k}$ is bijective. This gives, for each $\mathbf{i}$, a parametrization of $\mathbf{B}$ by elements of $\mathbb{N}^{k}$.

Lusztig introduced [6] two particular reduced words $\mathbf{j}, \mathbf{j}^{\prime}$ for $w_{0}$. In type $A_{n}$ these are as follows. Suppose the vertices of the Dynkin diagram of $A_{n}$ are labelled as in Figure 2.

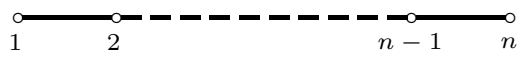

Figure 2: Dynkin diagram of type $A_{n}$. 
Let $\mathbf{j}$ be $135 \cdots 246 \cdots 135 \cdots 246 \cdots$, where $k=\frac{1}{2} n(n+1)$ factors are taken, and let $\mathbf{j}^{\prime}$ be $246 \cdots 135 \cdots 246 \cdots 135 \cdots$, where again $k$ factors are taken. Then $\mathbf{j}, \mathbf{j}^{\prime}$ are reduced words for $w_{0}$ and their commutation classes are as far apart as possible, in the sense that they give opposite orderings on $\Phi^{+}$. Consider the parametrizations $\varphi_{\mathbf{j}}: \mathbf{B} \rightarrow \mathbb{N}^{k}$ and $\varphi_{\mathbf{j}^{\prime}}: \mathbf{B} \rightarrow \mathbb{N}^{k}$ of $\mathbf{B}$ corresponding to $\mathbf{j}, \mathbf{j}^{\prime}$ and let $R=\varphi_{\mathbf{j}^{\prime}} \varphi_{\mathbf{j}}^{-1}: \mathbb{N}^{k} \rightarrow \mathbb{N}^{k}$ be the bijective map which relates them.

The function $R$ is the restriction to $\mathbb{N}^{k}$ of a piecewise-linear map $R: \mathbb{R}^{k} \rightarrow \mathbb{R}^{k}$ and the results in low rank cases suggest that the regions of linearity of $R$ are related to the different types of canonical basis elements.

For example, in type $A_{2}$, we have $\mathbf{j}=121, \mathbf{j}^{\prime}=212$, and $R$ has two regions of linearity. These correspond to the two types of monomial in the canonical basis.

In type $A_{3}$, the function $R$ has 10 regions of linearity, and 8 of these regions correspond to the 8 families of monomials in the canonical basis. The remaining 2 regions correspond to the remaining non-monomial elements of $\mathbf{B}$ obtained by Xi. These two regions may be distinguished from the other 8 regions as follows.

In type $A_{3}$, we have $R: \mathbb{R}^{6} \rightarrow \mathbb{R}^{6}$. The 8 regions of linearity of $R$ which give rise to monomials in $\mathbf{B}$ are each defined by 3 inequalities whereas the 2 remaining regions of linearity are each defined by 4 inequalities. This suggests that regions of linearity defined by the minimum number of inequalities might give rise to canonical basis elements of a particularly favourable form.

This turns out to be the case in type $A_{4}$ also. This time we have a piecewise-linear map $R: \mathbb{R}^{10} \rightarrow \mathbb{R}^{10}$, which was shown by Carter to have 144 regions of linearity. Of these regions, 62 are defined by 6 inequalities, 70 by 7 inequalities, 10 by 8 inequalities, and 2 by 11 inequalities. It is striking that the number of regions defined by the minimum number of inequalities is equal to the number of commutation classes of reduced words for $w_{0}$.

The authors have shown that in type $A_{4}$ there is a natural bijection between commutation classes of reduced words for $w_{0}$ and regions of linearity for $R$ defined by the minimum number of inequalities. This comes about as follows. For each reduced word $\mathbf{i}$ for $w_{0}$ we have a corresponding family $M_{\mathbf{i}}$ of monomials in $\mathbf{B}$, as described above. We consider the set of points in $\mathbb{N}^{10}$ which parametrize these elements of $\mathbf{B}$ under the map $\varphi_{\mathbf{j}}$ : let $X_{\mathbf{i}}^{+}=\varphi_{\mathbf{j}}\left(M_{\mathbf{i}}\right)$. Then one can show:

(a) $X_{\mathbf{i}}^{+}$is the set of all points in a region of linearity $X_{\mathbf{i}}$ of $R$ with coordinates in $\mathbb{N}$.

(b) $X_{\mathbf{i}}$ is a region defined by the minimum number of inequalities.

(c) The map $\mathbf{i} \rightarrow X_{\mathbf{i}}$ is a bijection between commutation classes of reduced words for $w_{0}$ and regions of $R$ defined by the minimum number of inequalities.

In fact, both the set of commutation classes of reduced words for $w_{0}$ and the set of regions of $R$ defined by the minimum number of inequalities can be given a natural graph structure, and the map $\mathbf{i} \rightarrow X_{\mathbf{i}}$ is then an isomorphism of graphs.

\section{Rectangle Calculus}

It is natural to ask whether the region $X_{\mathbf{i}}$ can be described in terms of $\mathbf{i}$ without using ideas concerned with the canonical basis, but simply in combinatorial terms. This can be done by a form of combinatorics which we call the rectangle calculus. The basic idea is to associate with the reduced word $\mathbf{i}$ a set of linearly independent vectors such that $X_{\mathbf{i}}$ is the set of all linear combinations of these vectors with non-negative coefficients. These vectors 
will be called spanning vectors of $X_{\mathbf{i}}$.

We first introduce the idea of a partial quiver. This is a Dynkin diagram with arrows on certain edges, such that the set of edges with arrows in non-empty and connected. An example of a partial quiver of type $A_{7}$ is given in Figure 3.

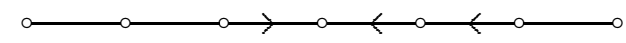

Figure 3: A partial quiver of type $A_{7}$.

We are concerned with partial quivers of type $A_{n}$, and shall use $L$ or $R$ to indicate whether an arrow goes left or right. Thus the above partial quiver is denoted $--R L L-$.

The possible partial quivers of type $A_{4}$ are $L L L, R L L, L R L, L L R, L R R, R L R, R R L, R R R, L L-,-L L, L R-$, $-L R, R L-,-R L, R R-,-R R, L--,-L-,--L, R--,-R-,--R$.

We shall now explain a procedure by which each reduced word $\mathbf{i}$ for $w_{0}$ in type $A_{n}$ gives a set of $\frac{1}{2} n(n-1)$ partial quivers. We first write down the braid diagram of $\mathbf{i}$. This determines a set of chambers, and for each bounded chamber we write down the corresponding chamber set, which is the subset of $\{1,2, \ldots, n+1\}$ corresponding to the strings which pass below the chamber. We illustrate this by the example in which $n=4$ and $w_{0}=$ $s_{2} s_{3} s_{4} s_{3} s_{1} s_{2} s_{1} s_{3} s_{2} s_{4}$ - see Figure 4 .

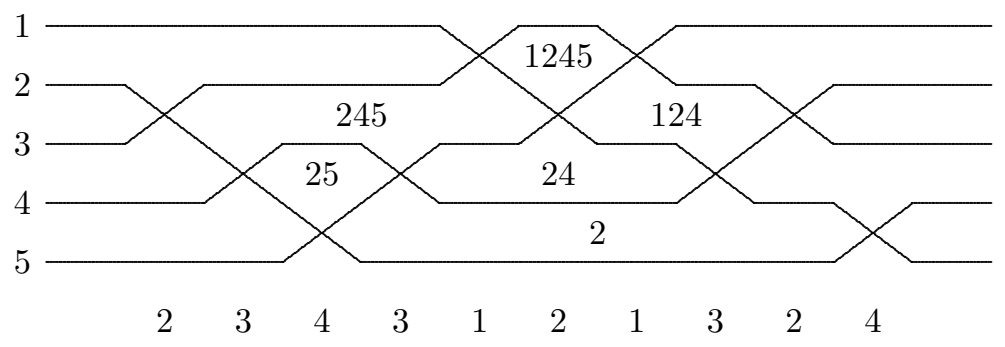

Figure 4: A chamber diagram.

Each chamber set obtained in this way is a subset of $\{1,2, \ldots, n+1\}$ which is not an initial or terminal subset, i.e. not of form $\{1,2, \ldots, i\}$ or $\{i, i+1, \ldots, n+1\}$ for any $i$.

Let $\mathcal{S}$ be the set of all subsets of $\{1,2, \ldots, n+1\}$ which are not initial or terminal subsets and $\mathcal{P}$ be the set of all partial quivers of type $A_{n}$. We shall describe a bijection from $\mathcal{S}$ to $\mathcal{P}$. We first number the edges of the Dynkin diagram as shown in Figure 5.

Thus the edges are numbered $2,3, \ldots, n$ from right to left and $1, n+1$ are regarded as virtual edges.

Let $S \in \mathcal{S}$ and $P$ be the corresponding partial quiver. $P$ is obtained from $S$ by the following rules. 


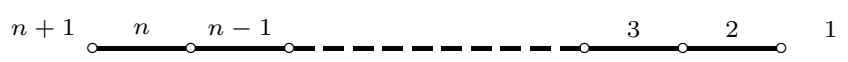

Figure 5: Edge numbering of Dynkin diagram.

(a) If $1, n+1 \notin S$, the entries in $S$ give the edges of type $L$ in $P$, edges intermediate between those of type $L$ having type $R$. The leftmost and rightmost labelled edges of $P$ have type $L$.

(b) Now suppose $1 \in S$. Let $i$ be such that $1,2, \ldots, i \in S$ but $i+1 \notin S$. Then edge $i+1$ is labelled $R$ and is the rightmost labelled edge in $P$.

(c) Now suppose $n+1 \in S$. Let $i$ be such that $i, i+1, \ldots, n+1$ lie in $S$ but $i-1 \notin S$. Then edge $i-1$ of $P$ is labelled $R$ and is the leftmost labelled edge in $P$.

(d) The elements of $S$ not in an initial segment as in (b) or a terminal segment as in (c) give rise to edges $L$ or $R$ in $P$ as in (a).

\section{Example.}

Let $n=13$. If $S=\{1,2,3,4,7,8,11\}$, then $P=--L R R L L R R---$. See Figure 6 for an explanatory diagram.

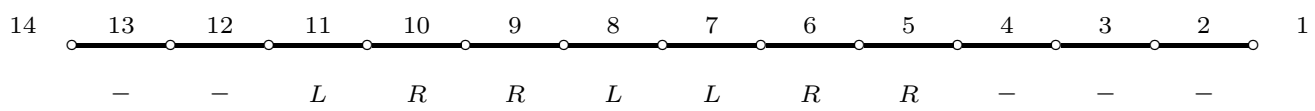

Figure 6: How to calculate the subset corresponding to a partial quiver.

Applying this bijection $\mathcal{S} \rightarrow \mathcal{P}$ to the chamber sets obtained from a reduced word $\mathbf{i}$ we obtain a set of $\frac{1}{2} n(n-1)$ partial quivers associated with $\mathbf{i}$. In the above example with $n=4$ and $w_{0}=s_{2} s_{3} s_{4} s_{3} s_{1} s_{2} s_{1} s_{3} s_{2} s_{4}$ we obtain the partial quivers shown in Figure 7.

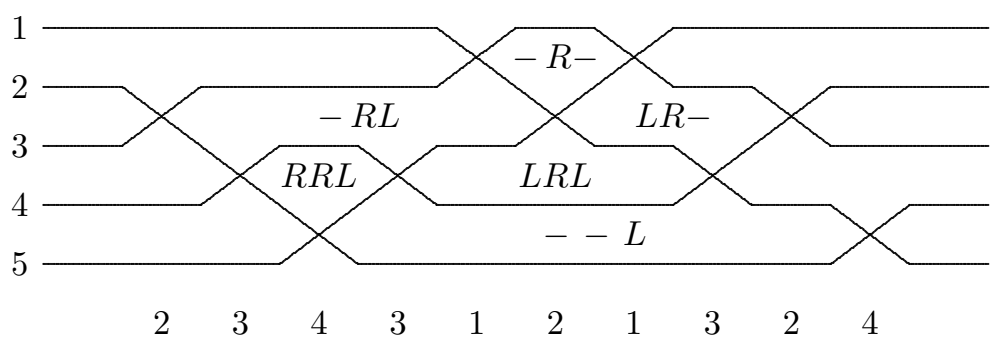

Figure 7: Partial quivers for $s_{2} s_{3} s_{4} s_{3} s_{1} s_{2} s_{1} s_{3} s_{2} s_{4}$.

We denote by $\mathcal{P}(\mathbf{i})$ the set of partial quivers obtained from $\mathbf{i}$ in this way. 
We now introduce the rectangles which we shall be considering. Let $i, j, k, l \in \mathbb{N}$ satisfy:

$$
i<j<l, \quad i<k<l, \quad i+l=j+k .
$$

An $(i, j, k, l)$-rectangle is a rectangle with corners on levels $i, j, k, l$. It is most convenient to illustrate this idea by means of an example. See Figure 8 .

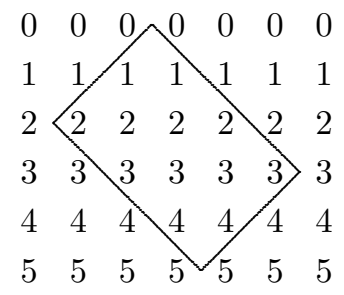

Figure 8: Drawing a $(0,2,3,5)$-rectangle.

The sides of the rectangle have gradient $\pm \pi / 4$. The $(i, j, k, l)$-rectangle contains alternate columns of integers starting with the first column if the entry in it is odd and the second column otherwise. See Figure 9.

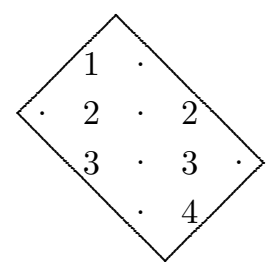

Figure 9: A $(0,2,3,5)$-rectangle.

The columns of integers in the rectangle are interpreted as positive roots; for example the $(0,2,3,5)$-rectangle contains roots $\alpha_{1}+\alpha_{2}+\alpha_{3}, \alpha_{2}+\alpha_{3}+\alpha_{4}$.

We next describe how each partial quiver determines a configuration of rectangles. It is again most convenient to explain this by means of an example. Consider the case of type $A_{10}$ in which we take the partial quiver $P=-L L R R R L R R$ - see Figure 10.

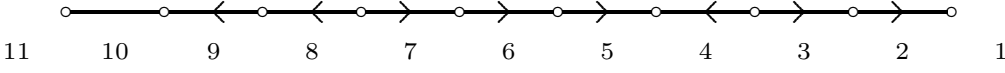

Figure 10: The partial quiver $P=-L L R R R L R R$.

The edges of the partial quiver are numbered as shown. We first divide the partial quiver into its components, i.e. the maximal subquivers containing a set of consecutive $L$ 's and $R$ 's. The components of our given partial 
quiver $P$ are:

$$
\begin{array}{cccccccccc}
- & L & L & - & - & - & - & - & - \\
- & - & - & R & R & R & - & - & - \\
- & - & - & - & - & - & L & - & - \\
- & - & - & - & - & - & - & R & R
\end{array}
$$

For each component $K$ of $P$ we define positive integers $a(K), b(K)$ with $a(K)<b(K)$. The integer $a(K)$ is the number of the edge following the rightmost arrow of $K$ and $b(K)$ is the number of the edge preceding the leftmost arrow of $K$. In the above example the numbers $a(K), b(K)$ are as follows:

\begin{tabular}{ccccccccccc}
\multicolumn{1}{c}{$K$} & & & & & $a(K)$ & $b(K)$ \\
- & $L$ & $L$ & - & - & - & - & - & - & 7 & 10 \\
- & - & - & $R$ & $R$ & $R$ & - & - & - & 4 & 8 \\
- & - & - & - & - & - & $L$ & - & - & 3 & 5 \\
- & - & - & - & - & - & - & $R$ & $R$ & 1 & 4
\end{tabular}

For each component $K$ of type $L$ we take a $(0, a, n+2-b, n+a-b+2)$-rectangle and for each component $K$ of type $R$ we take a $(b-a-1, b-1, n+1-a, n+1)$-rectangle, where $a=a(K), b=b(K)$. Thus the 4 components of our partial quiver $P=-L L R R R L R R$ give the 4 rectangles shown in Figure 11.

We now observe that for a component of type $L$ immediately followed by a component of type $R$ the two corresponding rectangles fit together from the left hand corner. Also, for a component of type $R$ immediately followed by a component of type $L$ the two rectangles fit together from the right hand corner. This can be observed in the four rectangles above, in which the first and the second fit from the left, the second and the third from the right, and the third and the fourth from the left.

We use these rules to superimpose the rectangles obtained from the components of the given partial quiver. In the case of the partial quiver $P=-L L R R R L R R$ we obtain the configuration of rectangles shown in Figure 12 .

We next define the centre of such a configuration. First consider the rectangles in the diagonals from north-west to south-east. The number of such rectangles in these diagonals in the above configuration is $1,3,4,2$. It is always the case that one obtains a set of odd numbers followed by a set of even numbers or vice versa. We draw the diagonal line separating the diagonal blocks giving odd and even numbers of rectangles. This is the line $\ell$ in Figure 13. A similar phenomenon occurs for the diagonals from northeast to southwest. The number of rectangles in such diagonals in the above configuration is $2,4,3,1$ and we draw the diagonal line separating the diagonal blocks giving odd and even numbers of rectangles. This is the line $\ell^{\prime}$ in Figure 13. The point $O$ in which $\ell$ and $\ell^{\prime}$ intersect is called the centre of the configuration and we draw in the vertical line through $O$, called the central line, $m$. See Figure 13 .

We consider the left and right hand corner points in this configuration. These are labelled $A, B, C, D, E$ in the above example. To each such corner point we associate the rectangle which has the given point as a vertex and 


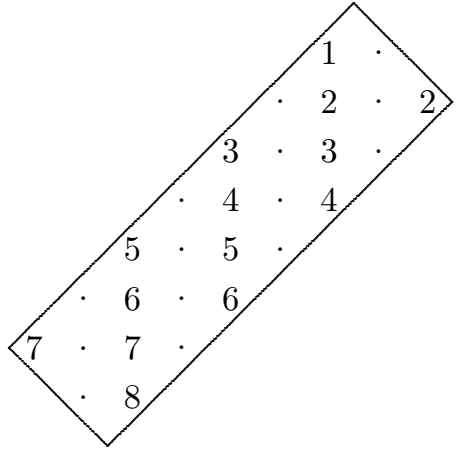

$-L L-----$

A $(0,7,2,9)$-rectangle

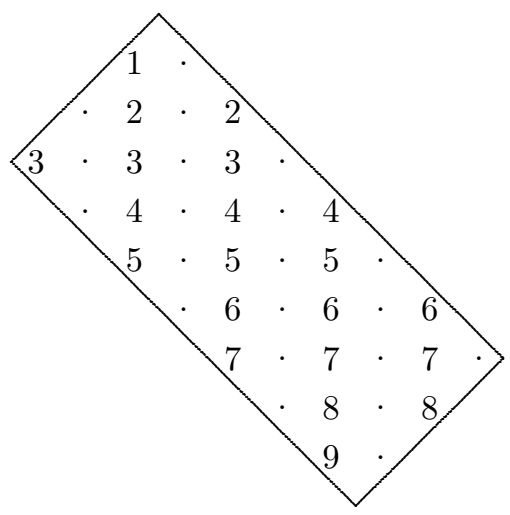

$-----L--$

A $(0,3,7,10)$-rectangle

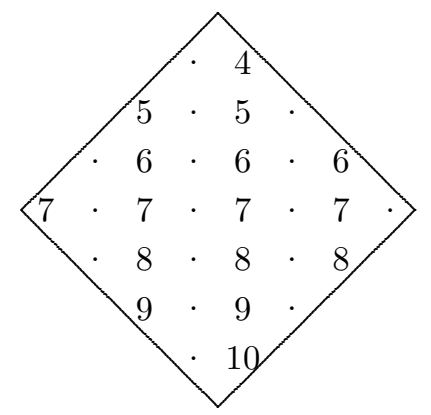

$---R R R---$

A $(3,7,7,11)$-rectangle

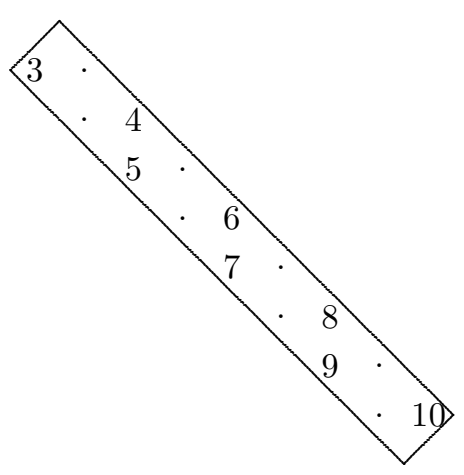

$------R R$

A $(2,3,10,11)$-rectangle

Figure 11: The 4 rectangles for $-L L R R R L R R$.

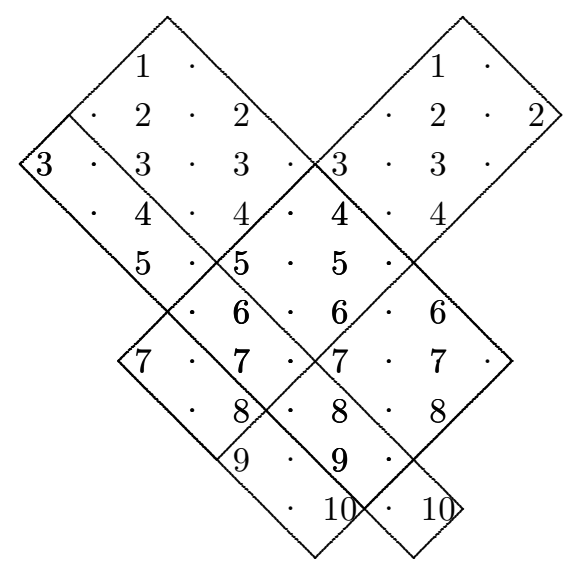

Figure 12: Configuration of all 4 rectangles. 


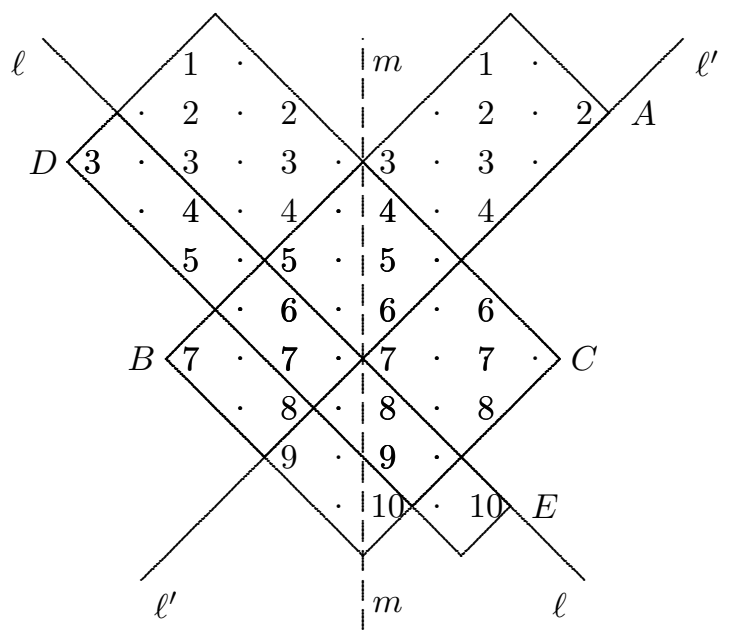

Figure 13: Configuration of all 4 rectangles with central line.

whose edges through this point extend as far as possible in the figure. (The vertex of the rectangle opposite to the given corner point may not be explicitly shown in the figure). For each such corner point $V$ we define a set of positive roots $\Phi^{+}(V)$. This is the set of all positive roots in the rectangle associated with $V$ on the same side of the central line $m$ as $V$ itself.

Thus in the given example, we have:

$$
\begin{aligned}
\Phi^{+}(A) & =\left\{\alpha_{2}, \alpha_{1}+\alpha_{2}+\alpha_{3}+\alpha_{4}, \alpha_{3}+\alpha_{4}+\alpha_{5}+\alpha_{6}\right\} \\
\Phi^{+}(B) & =\left\{\alpha_{7}, \alpha_{5}+\alpha_{6}+\alpha_{7}+\alpha_{8}+\alpha_{9}\right\} \\
\Phi^{+}(C) & =\left\{\alpha_{6}+\alpha_{7}+\alpha_{8}, \alpha_{4}+\alpha_{5}+\alpha_{6}+\alpha_{7}+\alpha_{8}+\alpha_{9}+\alpha_{10}\right\} \\
\Phi^{+}(D) & =\left\{\alpha_{3}, \alpha_{1}+\alpha_{2}+\alpha_{3}+\alpha_{4}+\alpha_{5}, \alpha_{2}+\alpha_{3}+\alpha_{4}+\alpha_{5}+\alpha_{6}+\alpha_{7}\right\} \\
\Phi^{+}(E) & =\left\{\alpha_{10}, \alpha_{8}+\alpha_{9}\right\}
\end{aligned}
$$

We then define $\Phi^{+}(P)$ to be the union of the sets $\Phi^{+}(V)$ for all corner points $V$ in the configuration. This is always a disjoint union. In the given example we have:

$$
\begin{aligned}
\Phi^{+}(P)= & \left\{\alpha_{2}, \alpha_{1}+\alpha_{2}+\alpha_{3}+\alpha_{4}, \alpha_{3}+\alpha_{4}+\alpha_{5}+\alpha_{6}, \alpha_{7}, \alpha_{5}+\alpha_{6}+\alpha_{7}+\alpha_{8}+\alpha_{9},\right. \\
& \alpha_{6}+\alpha_{7}+\alpha_{8}, \alpha_{4}+\alpha_{5}+\alpha_{6}+\alpha_{7}+\alpha_{8}+\alpha_{9}+\alpha_{10}, \alpha_{3}, \alpha_{1}+\alpha_{2}+\alpha_{3}+\alpha_{4}+\alpha_{5}, \\
& \left.\alpha_{2}+\alpha_{3}+\alpha_{4}+\alpha_{5}+\alpha_{6}+\alpha_{7}, \alpha_{10}, \alpha_{8}+\alpha_{9}\right\} .
\end{aligned}
$$

We now define a vector $v_{P} \in \mathbb{N}^{k}$ whose coordinates are all 0 or 1 . Let $\mathbf{j}$ be the reduced word

$$
135 \cdots 246 \cdots 135 \cdots 246 \cdots,
$$

considered above, and let $\alpha^{1}, \alpha^{2}, \ldots, \alpha^{k}$ be the corresponding order on the set of positive roots. We define $v_{P}$ as the vector whose $i$ th coordinate is 1 if $\alpha^{i} \in \Phi^{+}(P)$ and is 0 otherwise. We also define vectors $v_{j} \in \mathbb{N}^{k}$ for $j=1,2, \ldots, n$, where the $i$ th component of $v_{j}$ is 1 if the $i$ th letter in $\mathbf{j}$ is $j$ and is 0 otherwise.

These vectors $v_{P}, P \in \mathcal{P}(\mathbf{i})$ and $v_{j}, j \in\{1,2, \ldots, n\}$ turn out to be our required spanning vectors for the region $X_{\mathbf{i}}$ 
Proposition 3.1 Suppose that $\mathbf{g}$ has type $A_{4}$ and let $\mathbf{j}$ be the reduced word 1324132413 for $w_{0}$. Let $\mathbf{i}$ be any reduced word for $w_{0}$ and $\mathcal{P}(\mathbf{i})$ be the set of partial quivers associated with $\mathbf{i}$. We have $|\mathcal{P}(\mathbf{i})|=6$. Then the region $X_{\mathbf{i}}^{+}$associated with $\mathbf{i}$ is the set of all non-negative integral combinations of the vectors $v_{P}, P \in \mathcal{P}(\mathbf{i})$, and $v_{j}$ for $1 \leq j \leq 4$.

This Proposition explains how the regions $X_{\mathbf{i}}^{+}$can be described by the rectangle combinatorics.

\section{Speculations for type $A_{n}$}

It is natural to ask whether the set $X_{\mathbf{i}}$ defined as the set of non-negative linear combinations of the vectors $v_{P}$, $P \in \mathcal{P}(\mathbf{i})$ and $v_{j}, 1 \leq j \leq n$ is a region of linearity for $R: \mathbb{R}^{k} \rightarrow \mathbb{R}^{k}$ in type $A_{n}$. No counter-example is known to the authors. If so, are the $X_{\mathbf{i}}$ the only regions of linearity of $R$ defined by the minimum number of inequalities? This would give a bijection in type $A_{n}$ between commutation classes of reduced words for $w_{0}$ and regions of linearity for $R$ defined by the minimum number of inequalities.

The set $M_{\mathbf{i}}$ of monomials corresponding to points in the Lusztig cone $C_{\mathbf{i}}$ is not in general contained in the canonical basis in type $A_{n}$. It is nevertheless possible to consider a subset of $\mathbf{B}$ in bijective correspondence with $C_{\mathbf{i}}$ by means of Kashiwara's approach to the canonical basis. Kashiwara [3] defines certain root operators $\widetilde{F}_{\mathbf{i}}$ which lead to a parametrization of the canonical basis B for each $\mathbf{i}$ by a certain subset $K_{\mathbf{i}} \subseteq \mathbb{N}^{k}$ which we call the string cone. This gives a bijection

$$
\psi_{\mathbf{i}}: \mathbf{B} \rightarrow K_{\mathbf{i}}
$$

It has been shown independently by Marsh [8] and by Premat [9] that $C_{\mathbf{i}} \subseteq K_{\mathbf{i}}$ for each i, i.e. that the Lusztig cone lies in the string cone. Thus we obtain a subset $\psi_{\mathbf{i}}^{-1}\left(C_{\mathbf{i}}\right) \subseteq \mathbf{B}$. This subset is equal to the set of monomials $M_{\mathbf{i}}$ when $n \leq 4$, but does not consist of monomials in general. Using Lusztig's parametrization

$$
\varphi_{\mathbf{j}}: \mathbf{B} \rightarrow \mathbb{N}^{k}
$$

where $\mathbf{j}=135 \cdots 246 \cdots 135 \cdots 246 \cdots$, this subset $\psi_{\mathbf{i}}^{-1}\left(C_{\mathbf{i}}\right)$ of $\mathbf{B}$ corresponds to a certain subset of $\mathbb{N}^{k}$. Let

$$
S_{\mathbf{i}}^{\mathbf{j}}: K_{\mathbf{i}} \rightarrow \mathbb{N}^{k}
$$

be the transition map given by $S_{\mathbf{i}}^{\mathbf{j}}=\varphi_{\mathbf{j}} \psi_{\mathbf{i}}^{-1}$. It is known that in type $A_{n}$, the images of the spanning vectors of the Lusztig cone $C_{\mathbf{i}}$ under $S_{\mathbf{i}}^{\mathbf{j}}$ are the vectors $v_{P}, P \in \mathcal{P}(\mathbf{i})$ and $v_{j}, 1 \leq j \leq n$ given by the rectangle combinatorics. This can be proved using a transition function introduced by Berenstein, Fomin and Zelevinsky [1].

Finally, what can be said about canonical basis elements corresponding under $\varphi_{\mathbf{j}}$ to regions of linearity of $R$ not defined by the minimum number of inequalities? The results of Xi [10] in type $A_{3}$ are interesting in this respect. In type $A_{3}$, Lusztig's function $R: \mathbb{R}^{6} \rightarrow \mathbb{R}^{6}$ has 10 regions of linearity, 8 of which are the regions $X_{\mathbf{i}}$ for the different commutation classes of reduced words $\mathbf{i}$ for $w_{0}$. These are all defined by 3 inequalities. The remaining two are defined by 4 inequalities and we denote these by $X_{9}$ and $X_{10}$. We now define $X_{\mathbf{i}}^{+}$to be the set of points in $X_{\mathbf{i}}$ whose coordinates are all real and nonnegative. Thus we have

$$
X_{\mathbf{i}}^{+} \subseteq \widetilde{X_{\mathbf{i}}^{+}} \subseteq X_{\mathbf{i}}
$$

and $X_{\mathbf{i}}^{+}$is the set of integral points in $\widetilde{X_{\mathbf{i}}^{+}}$. We define $\widetilde{X_{9}^{+}}$and $\widetilde{X_{10}^{+}}$similarly. We have additional inequalities defining $\widetilde{X_{\mathbf{i}}^{+}}$asserting that all coordinates are non-negative, but some of these inequalities will be redundant. In 
fact each of the 8 regions $\widetilde{X_{\mathbf{i}}^{+}}$can be defined by 6 inequalities and, for suitable numbering, $\widetilde{X_{9}^{+}}$can be defined by 8 inequalities and $\widetilde{X_{10}^{+}}$by 9 inequalities. The regions $\widetilde{X_{\mathbf{i}}^{+}}$are called simplicial regions as the number of defining inequalities is equal to the dimension of the ambient space. $\widetilde{X_{9}^{+}}$can be written as the union of two simplicial regions, and $\widetilde{X_{10}^{+}}$as the union of four simplicial regions.

Xi obtains 8 families of monomials in $\mathbf{B}$, which are parametrized by the integral points in the 8 simplicial regions $\widetilde{X_{\mathbf{i}}^{+}}$. In addition he obtains 6 families of elements in $\mathbf{B}$ which are not monomials. These are parametrized by the integral points in the two simplicial regions whose union is $\widetilde{X_{9}^{+}}$and the four simplicial regions whose union is $\widetilde{X_{10}^{+}}$. The canonical basis elements corresponding to a given region are all of the same type, i.e. they can all be expressed as a linear combination of monomials corresponding to a fixed reduced expression with quantum binomial coefficients and exponents lying on a line segment in $\mathbb{N}^{6}$.

The authors plan to give the proofs of the results described in this article in a forthcoming paper.

\section{References}

[1] A. Berenstein, S. Fomin, and A. Zelevinsky. Parametrizations of canonical bases and totally positive matrices. Adv. Math., 122(1):49-149, 1996.

[2] R. W. Carter and R. J. Marsh. Regions of linearity, Lusztig cones and canonical basis elements for the quantized enveloping algebra of type $A_{4}$. Preprint arXiv:math.QA/0008050.

[3] M. Kashiwara. On crystal bases of the $q$-analogue of universal enveloping algebras. Duke Math. J., 63(2):465$516,1991$.

[4] G. Lusztig. Canonical bases arising from quantized enveloping algebras. J. Amer. Math. Soc., 3:447-498, 1990.

[5] G. Lusztig. Introduction to quantized enveloping algebras. Progr. Math., 105:49-95, 1992.

[6] G. Lusztig. Introduction to Quantum Groups. Birkhäuser, Boston, 1993.

[7] R. J. Marsh. More tight monomials in quantized enveloping algebras. J. Alg., 204:711-732, 1998.

[8] R. J. Marsh. Rectangle diagrams for the Lusztig cones of quantized enveloping algebras of type A. Preprint arXiv:math.QA/0008040.

[9] A. Premat. The Lusztig cone and the image of the Kashiwara map. Private communication, 1999.

[10] N. Xi. Canonical basis for type $A_{3}$. Preprint, 1998. 